\title{
Optimal Positions Selection for Watermark Inclusion based on a Nature Inspired Algorithm
}

\author{
Jumana Waleed ${ }^{1,2}$, Huang Dong Jun ${ }^{1}$, Saad Hameed ${ }^{3}$ and May Kamil ${ }^{3}$ \\ ${ }^{1}$ School of information Science and Engineering, Central South University, \\ Changsha, 410083, China \\ ${ }^{2}$ College of Science, University of Diyala, Iraq \\ ${ }^{3}$ College of Information Science and Engineering, Hunan University, Changsha, \\ China \\ Jumana_waleed@yahoo.com,djhuang@csu.edu.cn,saad@hnu.edu.cn, \\ alazzawimay104@yahoo.com
}

\begin{abstract}
One of the powerful optimization tools that has been exploited in the computer world are nature inspired algorithms (NIAs), they are also used to solve problems in the computer programming world. For many years new algorithms have been developed regarding computer science and engineering communities such algorithms concentrates on NIAs which has proven their capabilities in many aspect, in some situation rapid solutions are needed to solve some problems these algorithms provides a versatile robust solutions for many of these situations. This paper presents a watermark inclusion based on a recently presented nature inspired algorithm to enhance the digital image watermarking procedure to be used for copyright protection. The nature inspired algorithm in focus is used to perfectly identify optimal positions in the discrete wavelet transform domain (DWT) for watermark inclusion in the gray scale image, The obtained results are shown in the experimental results section clarifying the superiority of using the algorithm in focus for the watermarking technique, In addition, showing how the algorithm optimum positions are obtained with lowest effect to the PSNR value of the produced watermark included images.
\end{abstract}

Keywords: Nature Inspired Algorithms (NIAs), Optimal Positions Selection

\section{Introduction}

Recently the most active and trending research filed is digital watermarking this is because of the rapid growth of digital multimedia and information representation. Thus a need for content protection is an essential requirement to ensure the ownership of that digital content.

Digital watermarking techniques can be classified into spatial domain and frequency domain according to the domain used for embedding watermark. Spatial domain based watermarking, focuses on modifying the pixels of one or two randomly selected subsets of images. It directly loads the raw data into the image pixels. Spatial domain has weak robustness against common image signal processing and attacks such as noise, filtering, and compression, and may be easily destroyed by distortion. Frequency domain based watermarking, this technique is also called transform domain, where the values of certain frequencies are altered from their original to another form. There are several common used transform domain methods, such as Discrete Wavelet Transform (DWT), Discrete Cosine Transform (DCT), Discrete Fourier Transform (DFT) and Singular Valued Decomposition (SVD). The process embeds a watermark into the selected portion of frequency domain by modifying the coefficients. The frequency domain based 
watermarking is known as more robust and imperceptible technique than the spatial domain based watermarking, so the frequency domain is mostly used in recent watermarking methods [1]. The DWT finds a great popularity in the field of watermarking as it is able to decompose the available images into sub bands, in which watermarks can be embedded [2].

Nowadays, NIAs have emerged as optimization algorithms for complex problems solving. In the application of digital image watermarking optimization, the uses of NIAs have helped in the complex task of finding the optimal positions and preferable parameters for watermark embedding. Two decades ago, many of new algorithms such as particle swarm optimization (PSO), differential evolution (DE), ant colony optimization (ACO), bee algorithm (BA), cat swarm optimization (CSO), firefly algorithm (FA) and cuckoo search (CS) have been developed and they have shown great potential in solving tough engineering optimization problems. This paper presents an optimized blind watermarking technique based on a new class of NIAs and modern approach for optimization purposes which was inspired by cuckoo brooding behavior for selecting the optimum positions to embed an imperceptible watermark in DWT domain with respectable level of robustness. The rest of the paper is organized as follows: section 2 explains the related works. Section 3 explains the NIA exploited in the proposed Technique. Section 4 describes the proposed optimal image watermarking based on NIA and the usage of the DWT domain. In Section 5, we present experiments to demonstrate the performance of the proposed technique and Section 6 concludes the work of this paper.

\section{Related Work}

For embedding the watermark in wavelet transform domain of the digital image, the low Frequency sub-band can easily be visible to human eye, and the high Frequency sub-band is more sensitive to compression and scaling operation. Even though the watermarks are embedded into the middle-frequency bands, the problem about how to choose the optimal embedding frequencies is still not solved. Optimal positions and strength parameters selection for watermark embedding are the most critical aspect in the whole watermarking process and it had attracted many researchers in the recent past. Recently, the natural evolution techniques that are found in biology have been employed to optimize the digital watermarking methods to solve these problems. In [3], the Genetic Algorithm (GA) is used to obtain the optimized location for watermark inclusion. While in [4], the watermark strength is intelligently selected through GA. A combined DWT and DCT watermarking scheme optimized using GA is proposed in [5]. For watermark embedding, the cover image was decomposed by a 2-level DWT, and the HL2 sub-band coefficient was divided into $4 \times 4$ blocks, then the DCT was performed on each of these blocks. GA is used to find which four middle bands of coefficient of block DCT are used to embed the watermark and to find the optimum value of gain factor. In [6], Particle Swarm Optimization (PSO) is used to select the best coefficient for embedding the watermark adaptively. While in [7], the strength of embedding watermark in different blocks is adaptive by invoking PSO algorithm. In [8], a new adaptive digital image watermarking method is proposed, in which DCT sub-band is selected using GA and watermark strength is intelligently selected through PSO. GA performs well at exploring a vast search space and it selects the suitable sub band for watermark embedding. Optimal DWT-SVD based image watermarking scheme using DE algorithm is presented in [9]. The role of DE algorithm is to search optimal scaling factors to improve the quality of watermarked image and robustness of the watermark. Also in [10], DE algorithm is used in a robust DCT-SVD based image watermarking scheme to find the suitable multiple scaling factors. Another 
evolutionary algorithm is used in [11] to present a novel fast digital image watermarking technique that takes inspiration from the Bee Algorithm (BA). An attempt has been made to retrieve watermark same as original watermark using Cat Swarm Optimization (CSO) technique is proposed in [12]. [13] Present a new watermarking algorithm based on Multi-Objective Ant Colony Optimization (MOACO) to find the set of watermarking scaling factors to achieve a highest possible robustness without losing watermark transparency. A novel image watermarking scheme using FA is proposed in [14] in which the location of the coefficients to be modified by the watermark are identified and selected by using FA.

Cuckoo search (CS) is a new class NIAs and modern approach for optimization purposes which was inspired by cuckoo brooding behaviour, because this algorithm is considered relatively new, it is neither been utilized in the field of digital watermarking nor evaluated as a watermark improvement scheme.

\section{The Proposed Watermarking Technique based on a NIA}

In the proposed embedding technique, the host image is decomposed in to four sub bands by using 1-level DWT, and the watermark bits are embedded in $L H, H L$ and $H H$ sub bands. These sub bands are modified based on CS algorithm. The proposed watermarking technique can be characterized as follows:

\subsection{Watermark Bits Embedding}

The watermark bits embedding process is described as follows:

- Reformulate the watermark image into a vector $W=\left\{w_{1}, w_{2}, \ldots, w_{m}\right\}$ of binaries.

- Apply 1-level DWT to decompose the host image $I$, in to four sub bands $L L, L H$, $H L$ and $H H$.

- Select $L H, H L$ and $H H$ sub bans and partition each one into non overlapped blocks of $8 \times 8$ pixels.

- For each watermark bit $W_{i}$ fetch an non repeated $8 \times 8$ block and identify its first horizontal coefficients $H_{c}$, its maximum coefficient MAXIMUM and its minimum coefficient MINIMUM, if the value of $W_{i}$ is 1 then the first element in $H_{c}$ is calculated by MAXIMUM $+\mu_{j}$, if the embedded bit equal to 0 the replacement is done by MINIMUM $-\mu_{j}$, where $\mu_{j}$ are the strength factor parameters $\left(\mu_{1}, \mu_{2}\right.$ and $\left.\mu_{3}\right)$ one for each sub band which are evaluated experimentally and chosen manually $\left(\mu_{1}=1.95, \mu_{2}=2\right.$ and $\left.\mu_{3}=2.55\right)$.

For each block $B_{i}$,

$$
B_{i}{ }^{\prime}(1,1)= \begin{cases}\operatorname{MAXIMUM}\left(H_{c}\right)+\mu_{j} & \text { if } W_{i}=1 \\ \operatorname{MINIMUM}\left(H_{c}\right)-\mu_{j} & \text { if } W_{i}=0\end{cases}
$$

- Perform the inverse DWT by including modified and non-modified blocks to get the watermarked image $I^{\prime}$.

- Measure the imperceptibility of the watermarked image by using the peak signal to noise ratio (PSNR). PSNR is defining as Equation (2).

$$
\text { PSNR }=10 \log _{10} \frac{255^{2}}{\text { MSE }}
$$

Where, the MSE is the mean squared error between the original and watermarked images, is defined as Equation (3). 


$$
\text { MSE }=\frac{1}{m \times m} \sum_{i=1}^{m} \sum_{j=1}^{m}\left[I(i, j)-I^{\prime}(i, j)\right]^{2}
$$

Where: $M \times M$ is the size of the image, and $I(i, j), I^{\prime}(i, j)$ are the pixel values of the host and the watermarked images. The larger PSNR is the less distortion caused by embedding watermark in the host image.

\subsection{Watermark Bits Extraction}

In watermark bits extraction process, the original host image is not required, and the process is done as follows:

- Apply 1-level DWT to decompose the watermarked image $I$ ', in to four sub bands $L L^{\prime}, L H^{\prime}, H L^{\prime}$ and $H H^{\prime}$.

- Use the positions that found with CS optimization algorithm in the embedding process as extraction key.

- In each sub bund, extract each watermark bit $W_{i}^{\prime}$ by computing the average $A V G$ of the first horizontal coefficients $H_{c}$ of each watermarked block, and if the first coefficient in $H_{c}$ is greater than the average then the watermark bit is equal to 1 , otherwise the watermark bit is equal to 0 .

For each block $B_{i}{ }^{\prime}$,

$$
W_{i}^{\prime}= \begin{cases}1, & \text { if } B_{i}^{\prime}(1,1) \geq A V G \\ 0, & \text { if } B_{i}^{\prime}(1,1)<A V G\end{cases}
$$

- Obtain the extracted watermark.

After obtaining the PSNR in the watermarked image, we are ready to start the NIA.

\section{NIA and its Implementation}

\subsection{Cuckoo Search (CS)}

CS was first proposed by Yang and Deb [15]. This algorithm is based on the obligate brood parasitic behaviour found in some cuckoo species and in the meantime combining the Lévy flight behaviour discovered in some birds and fruit flies. From a quick look, it seems that there is some similarity between CS and hillclimbing, in combination with some large scale randomization. But there are some significant differences that the authors summarized as follows: firstly, CS is a population-based algorithm, in a way similar to GA and PSO, but it uses some sort of elitism or selection similar to that used in harmony search; secondly, the randomization is more efficient as the step length is heavy-tailed, and any large step is possible; thirdly, the number of parameters to be tuned is less than GA and PSO, and thus it is potentially more generic to adapt to a wider class of optimization problems. In addition, each nest can represent a set of solutions; CS can thus be extended to the type of meta-population algorithm. For ease in describing CS, the three idealized rules are described as follows [15].

- Each cuckoo lays one egg at a time, and dumps its egg in randomly chosen nest;

- The best nests with high quality of eggs will carry over to the next generations;

- The number of available host nests is fixed, and the egg laid by a cuckoo is discovered by the host bird with a probability pa $\in[0,1]$. In this case, the host bird can either throw the egg away or abandon the nest, and build a completely new nest.

Based on these three rules, the basic steps of the CS can be summarized as the pseudo code as shown in Figure 1 [16]: 


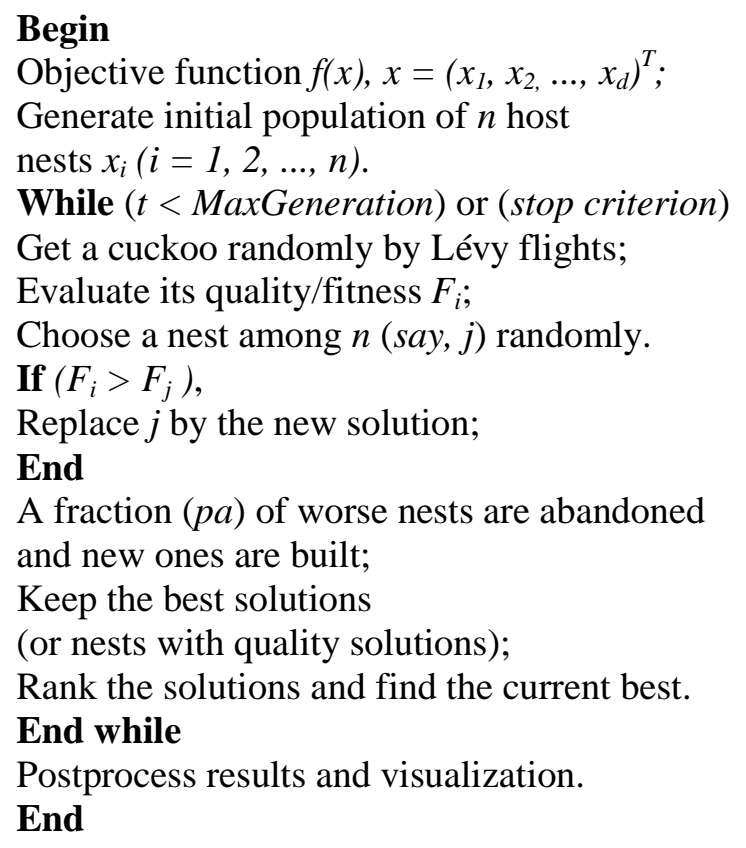

Figure 1. Cuckoo Search (CS)

Algorithmically, each host nest $n$ is defined as an agent which can contain a simple egg $x$ (unique dimension problem) or more than one, when the problem concerns to multiple dimensions.

When generating new solutions $x^{(t+1)}$ for a cuckoo, a Lévy flight is performed.

$$
x_{i}^{(t+1)}=x_{i}^{(t)}+\alpha \oplus \operatorname{Lévy}(\lambda)
$$

Where $\alpha>0$ is the step size which should be associated to the problem of interests scales, $\alpha$ can be set to value 1 in most situations. Equation (5) is basically the stochastic equation for random walk, which is a Markov chain whose next status or location only depends on the current status or location, and the transition probability, which are the first and second term respectively. The product $\oplus$ represents the entry wise multiplication, which is similar to those used in PSO. In terms of exploring the search space, random walk via Lévy flight is more efficient as its step length is much longer in the long run [15].

The random step length of Lévy flight, which fundamentally provides a random walk, is derived from a Lévy distribution with an infinite variance and infinite mean.

$$
\text { Lévy } \sim u=t^{-\lambda}, \quad(1<\lambda \leq 3)
$$

Here the steps essentially form a random walk process with a power-law steplength distribution with a heavy tail. Some of the new solutions should be generated by Lévy walk around the best solution obtained so far, this will speed up the local search. However, a substantial fraction of the new solutions should be generated by far field randomization and whose locations should be far enough from the current best solution [15]. Figure 2 shows an example of Lévy flight in a two dimensional plane. 


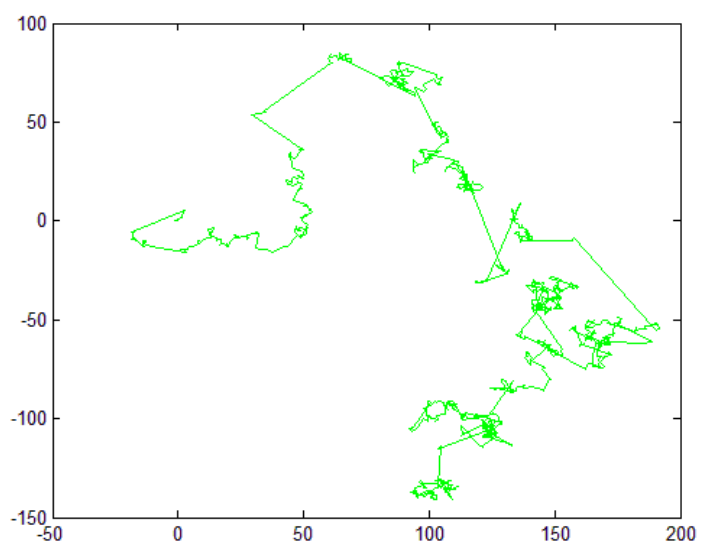

Figure 2. Example of Lévy Flight Starting at $[0,0]$

\subsection{NIA for Optimal Appropriate Blocks Positions Selection}

The main purpose for CS is to search for the proper blocks positions in the selected sub bunds $L H, H L$ and $H H$ for the watermark embedding, these blocks positions are used as a secret key. The CS is used considering the following steps:

1. Initialization of the CS parameters: the parameters for the CS algorithm consist of: number of nests $(n=60)$, nest size $=3072$, step size $(\alpha=1)$, number of generations $=1200$, and the probability to discover alien eggs $(\mathrm{pa}=0.25)$.

2. Generate initial nests: The CS is set to the population number $(n=60)$ and the initial range of host nests $=3072$. The initial nests will be evaluated using the objective function, which is the main contributor to CS.

3. Fitness Function: Peak Signal to Noise Ratio (PSNR) between the original and watermarked images is used as objective function to determine the quality of the solution.

4. Generation of cuckoo: A cuckoo is randomly generated by Lévy flight. The cuckoo is evaluated using the fitness function.

5. Replacement: a nest is selected among $n$ randomly, if the quality of new solution in the selected nest is better than the old solution, it will be replaced by the new solution (cuckoo). Note that all the nests except for the best one are replaced based on the quality of new cuckoo eggs.

6.Host discovery of cuckoo egg: The worse nests are abandoned based on the probability $(\mathrm{Pa}=0.25)$ and new ones are built.

7.Termination: In this paper, the termination criterion is set to 1200 generations. Once the number of training iterations in CS is reached, the optimization process is stopped. The nest with the highest fitness value in the final iteration is the optimized watermarked image. The optimized secret key for this image is sent to the receiver.

\section{Simulation Results}

To evaluate the performance of a NIA to select the optimal positions for the watermarking technique, standard gray scale images such as Lena, Cameraman, Peppers and Boat of size $512 \times 512$ have been used as host images shown in Figure $3(\mathrm{a}-\mathrm{d})$. A binary image of size $32 \times 32$ has been used as an imperceptible watermark shown in Figure 4. 


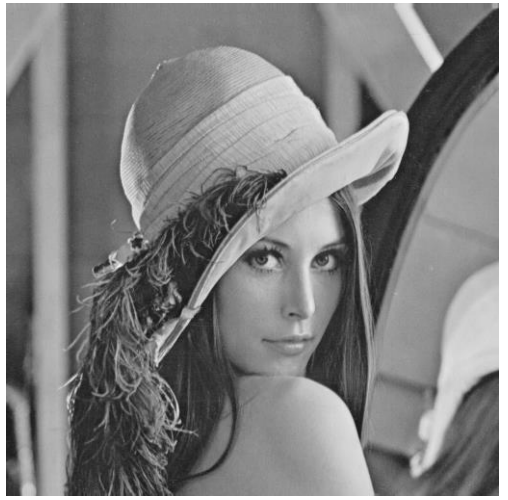

(a)

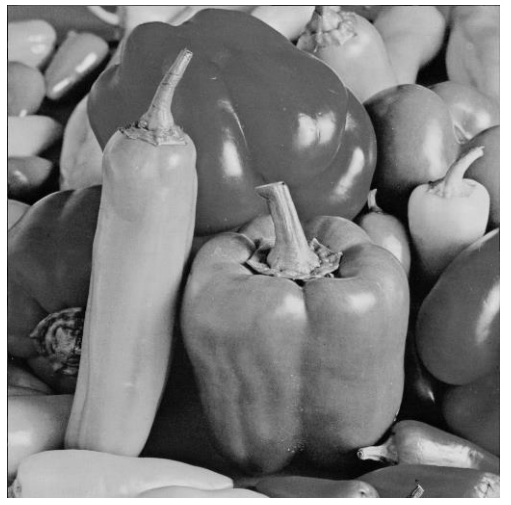

(c)

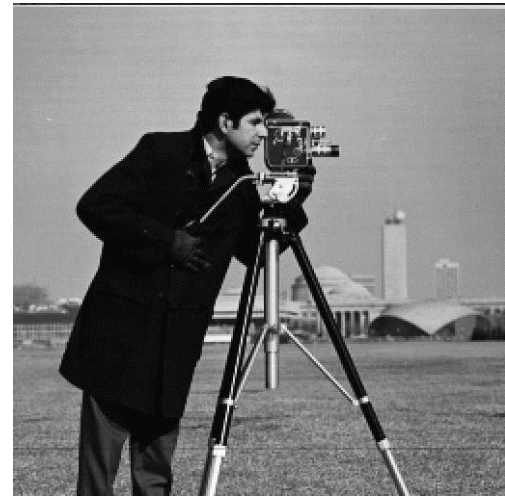

(b)

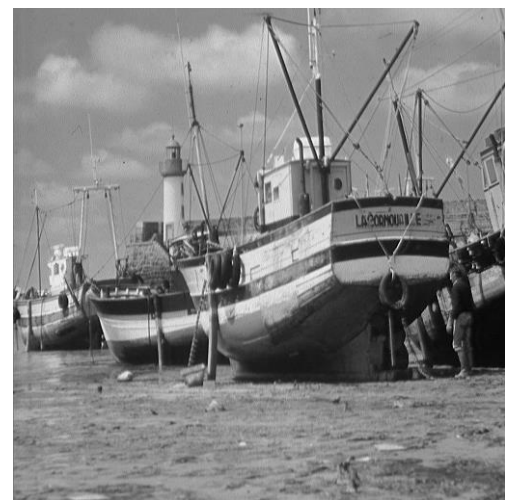

(d)

Figure 3. The Gray Scale Test Images (a) Lena, (b) Cameraman, (c) Peppers and (d) Boat

\section{CSU}

Figure 4. Watermark image $(32 \times 32)$.

In order to measure the robustness of the proposed watermarking system, the normalized correlation coefficient (NC) of the extracted watermark Wis is applied in conjunction to the original one Wi; the maximum value of this measure is 1 which determines the best robustness of the watermarking process.

$$
\mathrm{NC}=\frac{\sum_{i=1}^{\mathrm{N}} \mathrm{W}_{1} \mathrm{wi}_{1}^{\mathrm{x}}}{\sqrt{\sum_{i=1}^{\mathrm{N}} \mathrm{W}_{1}^{2}} \sqrt{\sum_{i=1}^{\mathrm{N}} \mathrm{Wi}_{1}^{\mathrm{T}_{2}}}}
$$

Where: $N$ is the size of the original and extracted watermark bits. Table 1 shows the $\mathrm{NC}$ values of the reconstructed watermark with random blocks positions selection for the proposed technique (without NIA optimization), and shows the NC values with the proposed optimization technique under different types of attack. Although the optimization process improved the values of PSNR it did not affect the values of NC under different types of attacks and sometimes improved the robustness. 
Table 1. The NC Values under Different Types of Attack with and without NIA Optimization

\begin{tabular}{|c|c|c|c|c|c|c|c|c|}
\hline \multirow{2}{*}{$\begin{array}{c}\text { Type of } \\
\text { attack }\end{array}$} & \multicolumn{4}{|c|}{ Without NIA optimization } & \multicolumn{4}{|c|}{ With NIA optimization } \\
\hline & Lena & Cameraman & peppers & Boat & Lena & Cameraman & peppers & Boat \\
\hline $\begin{array}{c}\text { Salt and } \\
\text { Pepper } \\
\text { Noise } 1 \%\end{array}$ & 0.9326 & 0.9260 & 0.9492 & 0.9457 & 0.9314 & 0.9240 & 0.9439 & 0.9421 \\
\hline $\begin{array}{l}\text { Salt and } \\
\text { Pepper } \\
\text { Noise 5\% }\end{array}$ & 0.8117 & 0.7797 & 0.8294 & 0.8599 & 0.8040 & 0.8051 & 0.8422 & 0.8465 \\
\hline $\begin{array}{l}\text { Gaussian } \\
\text { Noise }\end{array}$ & 0.9243 & 0.8779 & 0.9498 & 0.9545 & 0.9331 & 0.8922 & 0.9403 & 0.9415 \\
\hline $\begin{array}{c}\text { Median } \\
\text { Filter }(3 \times 3)\end{array}$ & 0.8014 & 0.8490 & 0.8003 & 0.7896 & 0.8139 & 0.8399 & 0.8201 & 0.8005 \\
\hline $\begin{array}{l}\text { Gaussian } \\
\text { LPF }(2 \times 2)\end{array}$ & 0.8325 & 0.8350 & 0.8282 & 0.8175 & 0.8274 & 0.8364 & 0.8454 & 0.8120 \\
\hline $\begin{array}{c}\text { Intensity } \\
\text { Adjustment }\end{array}$ & 0.9938 & 0.9966 & 0.9938 & 0.9960 & 0.9943 & 0.9945 & 0.9960 & 0.9977 \\
\hline $\begin{array}{c}\text { Gamma } \\
\text { Correction }\end{array}$ & 1.0000 & 0.9983 & 0.9989 & 1.0000 & 1.0000 & 0.9972 & 0.9989 & 1.0000 \\
\hline $\begin{array}{c}\text { Histogram } \\
\text { Equalization }\end{array}$ & 0.9972 & 0.9864 & 0.9955 & 0.9875 & 0.9977 & 0.9870 & 0.9976 & 0.9824 \\
\hline $\begin{array}{c}\text { Cropping } \\
30 \%\end{array}$ & 0.9785 & 0.9781 & 0.9812 & 0.9813 & 0.9808 & 0.9801 & 0.9780 & 0.9846 \\
\hline $\begin{array}{c}\text { JPEG } \\
\text { Compression }\end{array}$ & 0.8111 & 0.7694 & 0.8294 & 0.8450 & 0.8103 & 0.7819 & 0.8345 & 0.8339 \\
\hline
\end{tabular}

To investigate the robustness of the proposed technique, several attacks are applied to the watermarked images: Noisy attack is obtained by adding $1 \%$ and 5\% salt and pepper noise and adding Gaussian noise with mean $=0$ and variance $=0.0005$ to the watermarked image. Median filtering is performed with window size $3 \times 3$. And, Gaussian low pass filtering attack with a window size of $2 \times 2$ was applied to the watermarked images. Gamma Correction 1.5, Intensity Adjustment ([ $\left[\begin{array}{ll}0 & 0.8\end{array}\right]$, [ $\left[\begin{array}{ll}0 & 1\end{array}\right]$ ), and histogram equalization are applied to the watermarked image. The cropped image is obtained by cropping $30 \%$ of the watermarked image. JPEG compression attack is performed by compressing the image with quality factors 75 .

Figure 5 shows a clear climbing curve towards optimum results. The exact digital values of maximum fitness for the test images are 58.6320, 58.2305, 57.4446 and 56.0111 for Lena, Cameraman, Peppers and Boat respectively. Figure 6 represents the optimal blocks positions used for watermarking based on a NIA optimization for the gray scale test images. 


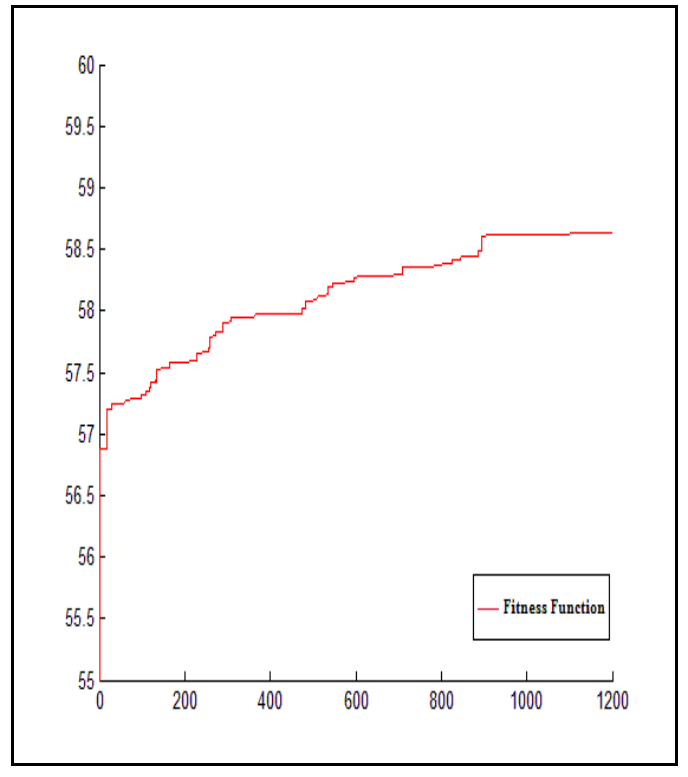

(a)

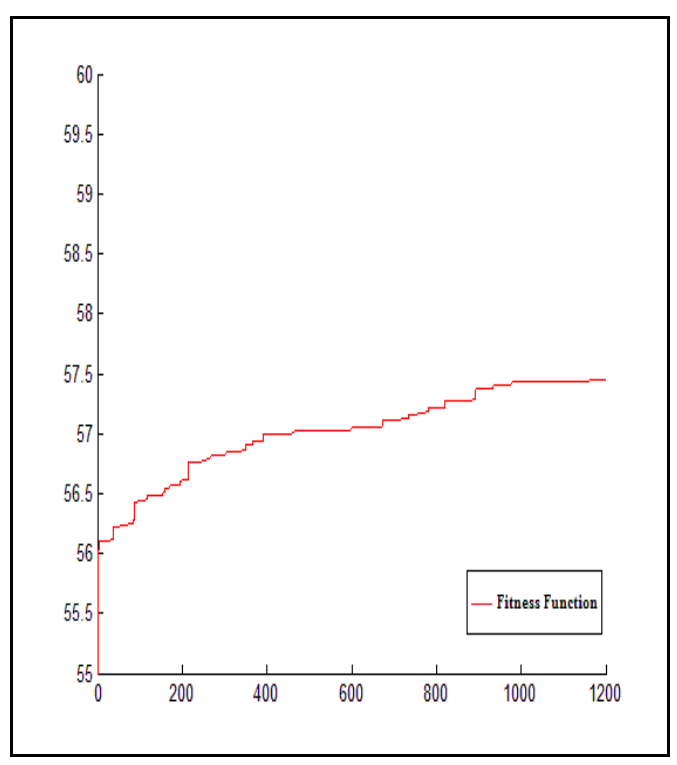

(c)

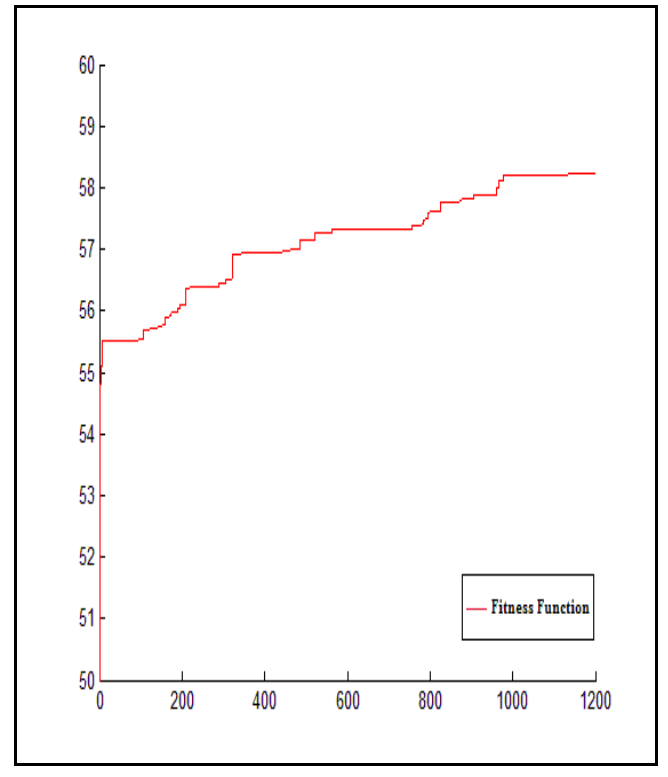

(b)

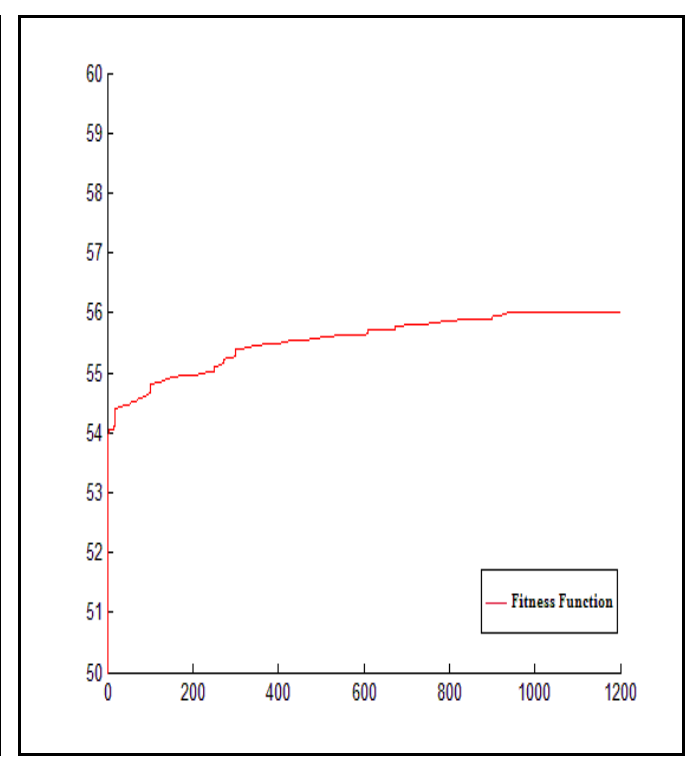

(d)

Figure 5. The Fitness Function Values Versus CS generations for (a) Lena, (b) Cameraman, (c) Peppers and (d) Boat Test Images 


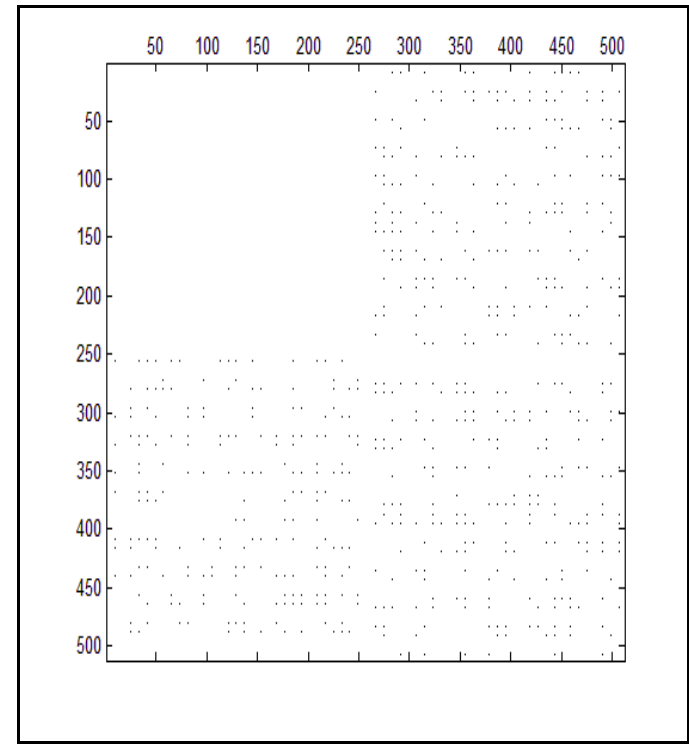

(a)

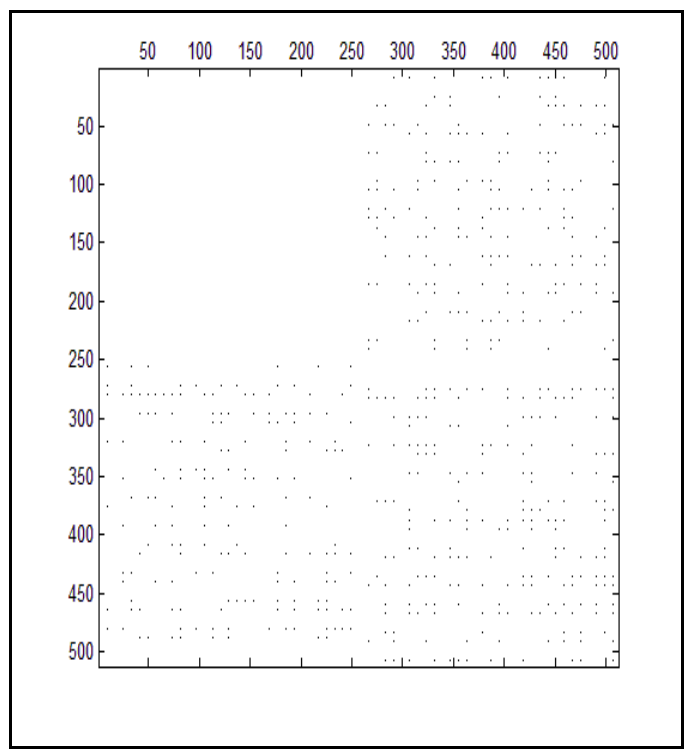

(c)

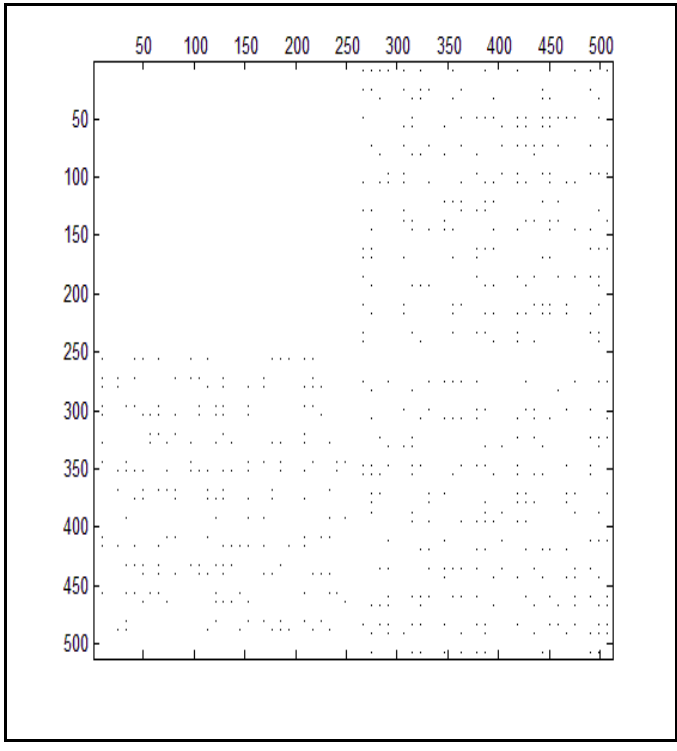

(b)

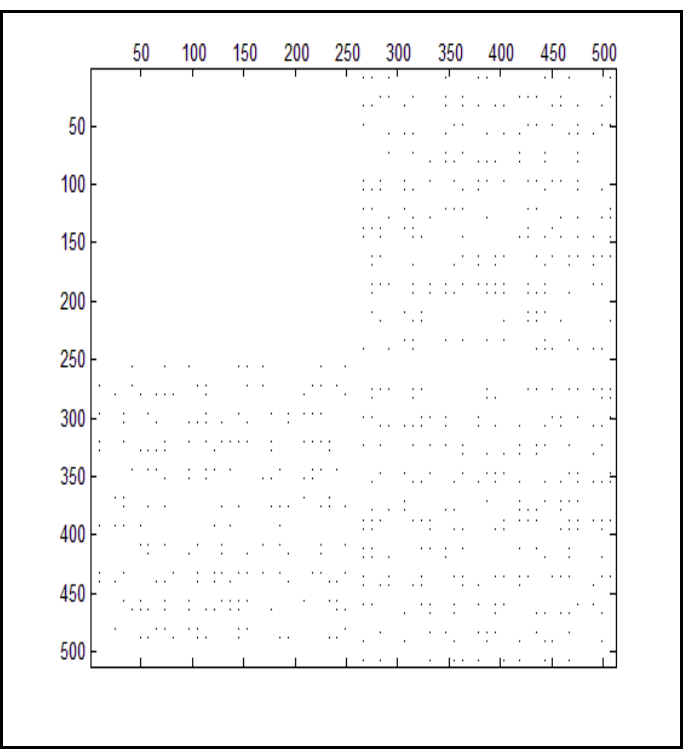

(d)

Figure 6. The Optimal Blocks Positions used for Watermarking based on CS Optimization for (a) Lena, (b) Cameraman, (c) Peppers and (d) Boat Test Images

Table 2 shows the values of all PSNR values obtained through the passing generations of the CS algorithm in contrast with PSNR values of a watermarking technique lacking the CS optimization ability. Table 3 shows a comparison in PSNR values for the watermarked Lena image and $\mathrm{NC}$ values for the extracted watermark without attacks between the proposed technique and the existing techniques [3, 5, 8, 11]. 
Table 2. Comparison of the Imperceptibility between the Proposed NonOptimized and Optimized Watermarking Techniques

\begin{tabular}{|c|c|c|c|}
\hline Test images & $\begin{array}{l}\text { Without optimization } \\
\text { (random positions) }\end{array}$ & $\begin{array}{c}\text { No. of } \\
\text { generations }\end{array}$ & $\begin{array}{l}\text { With optimization based on } \\
\text { NIA }\end{array}$ \\
\hline \multirow{6}{*}{ Lena } & \multirow{6}{*}{55.5056} & 200 & 57.5755 \\
\hline & & 400 & 57.9820 \\
\hline & & 600 & 58.2654 \\
\hline & & 800 & 58.3658 \\
\hline & & 1000 & 58.6114 \\
\hline & & 1200 & 58.6320 \\
\hline \multirow{6}{*}{ Cameraman } & \multirow{6}{*}{53.4768} & 200 & 56.1067 \\
\hline & & 400 & 56.9362 \\
\hline & & 600 & 57.3161 \\
\hline & & 800 & 57.5740 \\
\hline & & 1000 & 58.2064 \\
\hline & & 1200 & 58.2305 \\
\hline \multirow{6}{*}{ Peppers } & \multirow{6}{*}{54.9713} & 200 & 56.5970 \\
\hline & & 400 & 56.9937 \\
\hline & & 600 & 57.0341 \\
\hline & & 800 & 57.2107 \\
\hline & & 1000 & 57.4270 \\
\hline & & 1200 & 57.4446 \\
\hline \multirow{6}{*}{ Boat } & \multirow{6}{*}{52.8991} & 200 & 54.9532 \\
\hline & & 400 & 55.4751 \\
\hline & & 600 & 55.6425 \\
\hline & & 800 & 55.8751 \\
\hline & & 1000 & 56.0111 \\
\hline & & 1200 & 56.0111 \\
\hline
\end{tabular}

Table 3. Comparison between the Proposed Optimized Watermarking Technique and other Techniques

\begin{tabular}{|c|c|c|c|c|c|}
\hline Performance & $\begin{array}{c}\text { Huang et } \\
\text { al. [3] }\end{array}$ & $\begin{array}{c}\text { Mingzhi et } \\
\text { al. [5] }\end{array}$ & $\begin{array}{c}\text { Chaudhry et } \\
\text { al. [8] }\end{array}$ & $\begin{array}{c}\text { Farhan and } \\
\text { Bilal [11] }\end{array}$ & Proposed \\
\hline PSNR & $40.71 \mathrm{~dB}$ & $41.52 \mathrm{~dB}$ & $48.10 \mathrm{~dB}$ & $40.71 \mathrm{~dB}$ & $58.63 \mathrm{~dB}$ \\
\hline NC & 0.96 & 1.00 & 0.99 & 0.98 & 1.00 \\
\hline
\end{tabular}

\section{Conclusions}

In this paper a new optimized watermarking technique to select the optimal positions for watermark inclusion based on a NIA is proposed, tested and evaluated. The technique uses a recently presented NIA to improve the performance of watermarking, a nest is represented as a sequence of random locations (initially) and optimizing these sequences in the CS optimization algorithm to find the best nest with maximization effect on the PSNR (Fitness function). This technique presented noticeable improvements over successive generations. The outcome of the improvement technique is the preferable positions in the discrete wavelet transform domain of the gray scale images for the watermark embedding which used as an associated secret key.

It is very clear that image quality has been improved dramatically even when a watermark is introduced inside it this improvement is achieved by CS optimization by 
choosing optimum embedding locations while keeping robustness at high levels. These results are shown clearly in table 2 , also presented in the earlier presented figures.

\section{Acknowledgements}

This work was supposed by the project of National Science Fund of China (No. 60873188).

\section{References}

[1] J. Waleed, H. D. Jun, T. Abbas, S. Hameed and H. Hatem, "A Survey of Digital Image Watermarking Optimization based on Nature Inspired Algorithms NIAs", International Journal of Security and Its Applications, vol. 8, no. 6, (2014), pp. 315-334.

[2] H. A. Abdallah, M. M. Hadhoud, A. A. Shaalan and F. E. A. El-Samie, "Blind Wavelet-Based Image Watermarking”, International Journal of Signal Processing, Image Processing and Pattern Recognition, vol. 4, no. 1, (2011), pp. 15-28.

[3] H. - C. Huang, C. - M. Chu and J. - S. Pan, "The Optimized Copyright Protection System With Genetic Watermarking", Soft Computing, vol. 13, no. 4, (2009), pp. 333-343.

[4] M. Mubeen, S. A. M. Gilani and K. Zafar, "Robust image watermarking in contourlet domain using multi objective genetic algorithm", IEEE Eighth International Conference on Digital Information Management (ICDIM), Islamabad, Pakistan, (2013) September 10-12, pp. 67-72.

[5] C. Mingzhi, L. Yan, Z. Yajian and L. Min, "A Combined DWT and DCT Watermarking Scheme Optimized Using Genetic Algorithm”, Journal Of Multimedia, vol. 8, no. 3, (2013), pp. 299-305.

[6] M. Rohani and A. N. Avanaki, "A watermarking method based on optimizing SSIM index by using PSO in DCT domain", Proceedings of the 14th IEEE International CSI Computer Conference, Tehran, Iran, (2009) October 20-21, pp. 418-422.

[7] Y. -R. Wang, W.-H. Lin and L. Yang, "An intelligent watermarking method based on particle swarm optimization”, Expert Systems with Applications, vol. 38, no. 7, (2011), pp. 8024-8029.

[8] A. Chaudhry, B. Sikandar and M. Ishtiaq, "Genetic Swarm Based Robust Image Watermarking", Proceedings of the 7th International Conference on Ubiquitous Information Management and Communication, ACM, Kota Kinabalu, Malaysia, (2013) January 17-19.

[9] M. Ali, C. W. Ahn and P. Siarry, "Differential evolution algorithm for the selection of optimal scaling factors in image watermarking", Engineering Applications of Artificial Intelligence, Special Issue: Advances in Evolutionary Optimization Based Image Processing, vol. 31, (2014), pp. 15-26.

[10] M. Ali, C. W. Ahn and M. Pant, "A robust image watermarking technique using SVD and differential evolution in DCT domain", Optik - International Journal for Light and Electron Optics, vol. 125, no. 1, (2014), pp. 428-434.

[11] A. A. Farhan and S. Bilal, "A novel fast and robust digital image watermarking using Bee Algorithm", IEEE 14th International Multitopic Conference (INMIC), Karachi, Pakistan, (2011) December 22-24, pp. 82-86.

[12] G. Kalaiselvan, A. Lavanya and V. Natrajan, "Enhancing the Performance of Watermarking Based on Cat swarm Optimization Method", IEEE International Conference on Recent Trends in Information Technology (ICRTIT), Chennai, Tamil Nadu, (2011) June 3-5, pp. 1081-1086.

[13] K. Loukhaoukha, J.-Y. Chouinard and M. H.Taieb, "Optimal Image Watermarking Algorithm Based on LWT-SVD via Multi-objective Ant Colony Optimization", Journal of Information Hiding and Multimedia Signal Processing, vol. 2, no. 4, (2011), pp. 303-319.

[14] C. Agarwal, A. Mishra, A. Sharma and G. Chetty, "A Novel Image Watermarking Scheme using firefly Algorithm”, International Conference on Artificial Intelligence and software Engineering, (2014), pp. 430-436.

[15] X. S. Yang and S. Deb, "Cuckoo Search via L'evy Flights", IEEE World Congress on Nature \& Biologically Inspired Computing, Coimbatore, India, (2009) December 9-11, pp. 210-214.

[16] X. S. Yang and S. Deb, "Engineering Optimisation by Cuckoo Search", Int. J. Mathematical Modelling and Numerical Optimisation, vol. 1, no.4, (2010), pp. 330-343. 


\section{Authors}

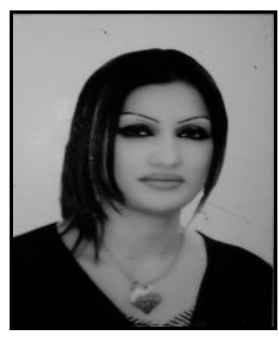

Jumana Waleed is a Ph.D. student in the School of information science and Engineering at Central South University in P.R. China. Her research activity focuses on image processing, and information security working on digital watermarking. She received the B.S. degree in computers sciences from the AlYarmouk University College, Iraq, in 2004, and the M.S. degree in Computer Science/Data Security from the University of Technology, Baghdad, Iraq, in 2009.

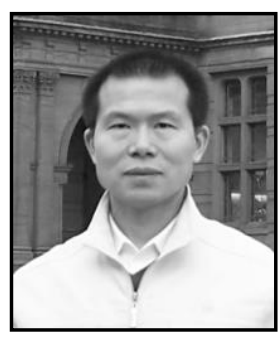

Dr. Huang Dong Jun, is a professor at Central South University. He received his PHD degree in computer science and technology from the Central South University, China, in 2004. He worked as a visiting academic to University of Glasgow, UK, from 2007 to 2008. Currently, he is the director of the Department of Computer Engineering, Central South University and the member of the IOT Education Expert Group of the Ministry of Education, China. His research interests include computer networks, multimedia technology, image and video processing, video conferencing system and video surveillance techniques.

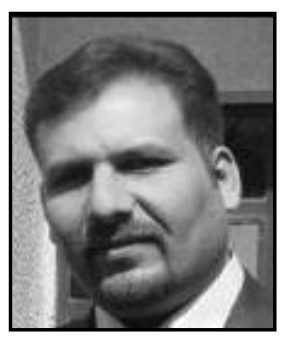

Saad Hameed was born in Baghdad - Iraq in 7 June 1979, he received his B.Sc. Degree in computer science at AL-Mansour University College 2001, and Masters Degree in computer Sciences, Iraqi committee for computer and informatics in 2004, he continued working in academic teaching in AL-Mansour University College for 11 years, through that time all of his research was self-funded and concentrated in automation and control, he has been promoted from assistant Lecturer to Lecturer in 2011, he is now a Ph.D. degree student at Hunan University in P.R. China.

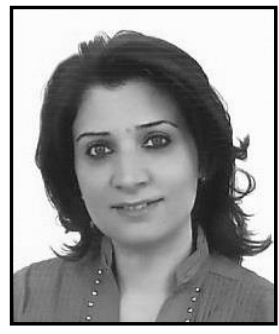

May Kamil received her B.Sc. degree in computer Science from Baghdad University, Iraq, 1993, and the M.Sc. in computer Science from University of Technology, Baghdad, Iraq, 2001, currently she is a Ph.D. Student in Hunan University, Changsha, China. 
International Journal of Signal Processing, Image Processing and Pattern Recognition Vol. 8, No. 1 (2015) 